\title{
Plate IV.
}

Fig. 5. Large sori of the fungus on a carpellary leaf. Nat. size.

Fig. 6. Cross section of the sori on a diseased leaf. Under the sori numerous accessory veins are produced. Slightly magnified.

Fig. 7. Cross section of a sorus through its peripheral portion. $\times 250$.

Fig. 8. Spermogonium. $\times 250$.

Fig. 9. Cross section of the cortex of an infected branch of the host. $m$, perennial mycelium. $h$, haustorium. $\times 350$.

Fig. 10. Normal leaf. Nat. size.

Fig. 11. Diseased leaf with enlarged stipules. Nat. size.

\section{On a New Species of Apocynaceæ from Formosa.}

by

\section{B. HAYATA.}

Assistant in the Botanical Institute, Science College, Imperial University of Trokyo.

The following interesting plant was kindly given to me by $\mathrm{Mr}$. T. Kawakami, Government Expert of Formesa. It was obtained by him at Shinko in the northern part of the island. He remarks that this tree is of great industrial value, on account of its milky juice which forms one of the kinds of Caoutchouc.

Ecdysanthera utilis Hayata et Kawakami. Frutices scandentes glabri. Folia opposita, elliptica, basi apiceque acuminata, 5-6 cm. longa, $3-3 \frac{1}{2} \mathrm{~cm}$. lata, subtus pallida remote pennivenia, venis utrinque $5-6$, longe petiolata, petiolis 2-3 cm. longis. Cyms laxce pubescentes, trichotomæ, ad apices ramorum paniculatæ, $7-8 \mathrm{~cm}$. longæ. Flores parvi, patentes $3 \mathrm{~mm}$. in diametro æquantes, flavescentes. Calyx parvus $1 \mathrm{~mm}$. longus, extus pubescens, 5-fidus, lobis triangularibus obtusis, basi intus glandulosus, glandulis brevibus cum lobis calycis alternis. Corolla suburceolata, tubo $1.5 \mathrm{~mm}$. longo ovoideo-campanulato, fauce intus pubescenti collosis cum lobis alternis; lobis 5, rotundato-truncatis basi breve angustis $1 \mathrm{~mm}$. latis brevibus leviter mucronatis latisque contortis dextrorsum obtegentibus. Stamina basi corolle affixa, inclusa, filamentis brevibus ; anthere lanceolatosagittatæ, $1 \frac{1}{2} \mathrm{~mm}$. longæ, acuminatæe, circa stigma conniventes, loculis basi in appendiculas vacuas longas productis. Discus annularis, 5-lobus. Ovarii 
carpella 2, distineta, disco longiora; stylus brevis; stigma ovoideum exannulatum, apiculo conico breviter 2-fido. Folliculi divaricati continui, teretes, $10 \mathrm{~cm}$. longi, $4 \mathrm{~mm}$. in sectione requantes. Semina in folliculo pauca, oblonga, $11 \mathrm{~mm}$. longa, $3 \mathrm{~mm}$. lata, compressa, a pice parum contracta ; coma $4 \mathrm{~cm}$. longa caduca et annulo pilorum brevium persistente coronata; albumen copiosum ; cotyledones oblonga, foliacere ; radicula longa, cotyledones in longituline requans.

Hab. Shinkō, Shintiku, leg. 'T. Kawakami, anno 1906 (Mart. Fl.).

It was suggested to me by Mr. T. Kawakam that this species may belong to Urceola. Studying this point carefully, I think that the plant should be referred to Ecdysanthera on account of its glands within the sepals, and its lobes of the corolla being imbricate. This new species resembles E. densiflora Miq. (Fl. Ind. Bat. II. p. 4.52); but differs from it by the long petioled leaves and flowers.

\title{
Contributions to the Flora of Mt. Morrison.
}

by

\author{
B. HAYATA. \\ Assistant in the Botanical Institute, Science College, \\ Imperial University of Tokiy $\overline{\text { o. }}$
}

Bœhnninghausenia albiflora ReICH. "Conspect. Reg. Veg. p. 197"; Hook. f. Fl. Brit. Ind. I. p. 486 ; Hance, in Journ. Bot. (1874) p. 259 ; Franchet, Pl. David. p. 66 ; Franch. et Sav. Enum. Pl. Jap. I. p. 71 ; Miq. Prol. Fl. Jap. p. 209 ; Engl. in Eng. et Prant. Nat. Pfl. fam. III. -4, p. 130 ; Forbes et Hemse. Ind. Fl. Sin. I. p. 102 ; Diers, Fl. Centr. Chin. in Engt. Bot. Jahrb. XXIX. p. 423.

Hab. 'Tōhosha, ad 2930 ped., (No. 706); Tatsuhō, ad 3139 ped., (No. 716); Sanchōkei, ad 4000 ped., (No. 725), leg. S. Nagasawa, anno 1905. Nov.

Distrib. Monotype. Khasia, in the temperate regions of Himalaya; also in mountainous district of China and Japan, but not in any neighbouring country farther south than Formosa.

Paris lancifolia Hayata n. sp. Rhizoma... Canlis simplex, circ. $50 \mathrm{~cm}$, longus. Folia 7-8, arl apicem caulis verticillata, sessilia, lanceolato- 